\title{
Perfil microbiológico del saco herniario en hernias complicadas operadas en el Servicio de Urgencias*
}

\author{
Drs. JUAN L. MORALES G. ${ }^{1,2}$, LUIS CANALES F. ${ }^{1}$, Int. CARLOS MORALES A. ${ }^{3}$ RICARDO REBOLLEDO R. ${ }^{3}$, \\ JOSÉ L. NOVOA. ${ }^{3}$, EDUARDO OTERO V. ${ }^{3}$
}

1 Servicio de Cirugía, Hospital Clínico Herminda Martín, Chillán.

2 Docente, Universidad Católica de la Santísima Concepción.

3 Interno de Medicina, Universidad Católica de la Santísima Concepción. Chillán, Chile.

\begin{abstract}
Infection of hernia sac of complicated abdominal hernias

Background: When a complicated hernia is operated, there may be a higher risk of infection when a mesh is placed in a place potentially colonized by intestinal bacteria translocated from the gut. Aim: To perform a microbiological study of the hernia sac of patients subjected to emergency surgery due to complicated hernias. Material and Methods: Prospective study of patients operated for complicated abdominal hernias. Prior to the repair, a culture was obtained from the exposed sac. A portion of this sac was excised and cultured also. Results: Forty one patients aged 46 to 88 years (30 females) were studied. Only one sac tissue sample culture was positive. In six patients, the sac surface cultures disclosed coagulase negative Staphiloccocus. These patients did not receive antimicrobial therapy and although a mesh was placed, no complication was recorded. It is possible that these positive cultures were due to contamination of samples. Conclusions: From a microbiological point of view it is safe to place a mesh during the repair of complicated hernias
\end{abstract}

Key words: Complicated hernia, infection, mesh.

\section{Resumen}

La incarceración de una hernia abdominal es una de las emergencias quirúrgicas más comunes. La reparación protésica de las hernias complicadas está aún en discusión, ya que muchos cirujanos temen la infección de la malla al implantarla en un sitio inflamado y potencialmente colonizado por bacterias translocadas desde el tubo digestivo. Nuestro estudio tiene como objetivo el determinar la presencia de estos microorganismos en el saco herniario de los pacientes adultos operados de urgencia con diagnóstico de hernia abdominal complicada, a través de cultivos de la superficie y fragmento del mismo, junto con establecer una correlación con las variables clínicas y de laboratorio del paciente al ingreso. Se estudiaron 41 pacientes, observándose un cultivo positivo en la muestra de tejido del saco. Por otro lado, seis pacientes cuyas muestras de superficie resultaron con desarrollo de Staphiloccocus coagulasa negativo, a pesar de no utilizarse terapia antibiótica y haberse reparado con malla, presentaron una excelente evolución sin complicaciones ulteriores, por lo tanto, no descartamos que las muestras correspondan a falsos

*Recibido el 5 de Noviembre de 2008 y aceptado para publicación el 22 de Junio de 2009.

Correspondencia: Dr. Juan L. Morales G.

Francisco Ramírez s/n, Chillán, Chile.

E-mail: jlmoralesg@ucsc.cl 
positivos debido a contaminación de las mismas. Nuestra casuística permite aportar con un antecedente más al algoritmo de decisiones acerca de la utilización de prótesis en hernioplastía de urgencia. Nos parece que desde el punto de vista microbiológico local, la reparación protésica parece ser segura.

Palabras clave: Hernia, incarceración, prótesis.

\section{Introducción}

Desde la pasada década, la reparación quirúrgica de las hernias, ha sufrido dos cambios importantes: la asistencia laparoscópica y la utilización de material protésico ${ }^{1}$.

Estudios multicéntricos han demostrado la superioridad de la reparación protésica sin tensión sobre la sutura primaria, con disminución de las recurrencias en hasta un $75 \%{ }^{2,3}$. En Chile, la experiencia avala estos resultados con una tasa de recidiva que no supera el $1,1 \% 0^{4,5}$.

La incarceración de una hernia abdominal es una de las emergencias quirúrgicas más comunes ${ }^{6}$. Aproximadamente el 10\% de las hernias inguinales se presentan con esta complicación aguda, así como el $40 \%$ de las femorales, requiriendo cirugía de urgencia para su reparación. La posibilidad de que presente compromiso vascular (estrangulación) es de alrededor de un $0,29-2,9 \%{ }^{7,8}$. Se sabe que la cirugía de la hernia complicada presenta mayor mortalidad que la reparación electiva de una hernia no complicada, alcanzando tasas de 5 a 10 veces mayor 9 .

La utilización de material protésico en el lecho de una hernia complicada es controversial, y se discute aún si la contaminación del mismo, señala una contraindicación para la técnica ${ }^{10}$.

Diversos autores han propuesto que la incarceración y/o estrangulación de la hernia contribuye a la formación de edema del saco herniario ${ }^{11}$ y esto, junto a la obstrucción intestinal concomitante, facilitaría la translocación bacteriana a la región, especialmente de Echerichia coli ${ }^{12,13}$.

Lo anterior, determinaría un riesgo mayor de infección que puede alcanzar hasta a un $42 \%$, siendo la principal complicación de esta técnica ${ }^{14}$, pudiendo tener graves consecuencias para el paciente, entre ellos la pérdida de la malla, recidiva de la hernia o dolor abdominal crónico ${ }^{15}$, sin que la antibioticoterapia profiláctica haya demostrado efectividad significativa en su prevención ${ }^{16}$.

Son pocos los estudios publicados acerca del uso de malla en hernioplastía de urgencia, destacando en nuestro medio a Beltrán ${ }^{17}$, pero la mayoría de ellos han mostrado que no existe mayor diferencia en las complicaciones postoperatorias incluso en presencia de resección intestinal ${ }^{18-20}$.

El principal criterio para la elección del uso o no de prótesis en hernioplastia de urgencia es, la pre- ferencia del cirujano, al considerar las características clínicas del paciente y de las condiciones locales de la hernia, sin embargo, no existe consenso claro a este respecto.

No encontramos trabajos en la literatura que demuestren la presencia de microorganismos patógenos en el sitio de implantación de la malla en hernia complicada, y creemos importante su determinación, para aportar un antecedente más al algoritmo de decisiones a la hora de resolver estos cuadros clínicos.

Los objetivos de nuestro estudio son: determinar la presencia de agentes bacterianos en el saco herniario al momento de efectuar la reparación de urgencia de una hernia complicada (incarcerada o estrangulada), además de estimar las condiciones clínicas y/o de laboratorio que permitan de forma indirecta inferir la colonización bacteriana del saco herniario antes de efectuar su reparación quirúrgica en urgencias.

\section{Material y Método}

Se realizó un estudio prospectivo descriptivo que incluyó a los pacientes adultos, ingresados a través del servicio de urgencias, con diagnóstico de hernia complicada en cualquiera de sus variedades y que fueron reparadas quirúrgicamente en la unidad.

A dichos pacientes, previo a la reparación del defecto herniario y una vez expuesto el saco, se tomó muestra con tórula de su superficie (Muestra $\left.\mathrm{N}^{\circ} 1\right)$ y posteriormente, se resecó un segmento del mismo (Muestra $\mathrm{N}^{\circ} 2$ ). Ambas muestras se enviaron a cultivo.

Como criterios de inclusión al estudio se consideró a todo paciente mayor de 15 años con diagnóstico de hernia complicada (incarcerada, estrangulada) al que se le practique reparación quirúrgica del defecto en el servicio de urgencias del hospital clínico Herminda Martín de Chillán.

Definimos hernia incarcerada como aquella hernia que cursa con episodio agudo de dolor, tumefacción e irreductibilidad ante las maniobras de taxis.

Definimos hernia estrangulada a aquella hernia incarcerada en la que se comprueba compromiso vascular concomitante.

Fueron excluidos del estudio los pacientes menores de 15 años o aquellos en los que se eviden- 
Tabla 1. Variables estudiadas

Edad y sexo del paciente

Enfermedades concomitantes

Tipo de hernia y características anatómicas

Tipo de complicación y tiempo de evolución

Recuento de leucocitos y PCR preoperatorio

Tipo de reparación (Protésica o sutura simple)

Antibioticoterapia previa, profiláctica o terapéutica

ció real contaminación de la región comprometida, ya sea por necrosis extensa, perforación de víscera hueca, peritonitis u otra condición que impida la correcta toma del material de cultivo necesario.

Las variables a medir se resumen en la Tabla 1.

Los datos fueron recopilados a través de una ficha tipo completada por el cirujano o su ayudante una vez concluida la cirugía. Lo anterior se cotejó con la ficha clínica del paciente y el resultado del cultivo rescatado de la base de datos del laboratorio central. La información recogida fue tabulada en una planilla especialmente diseñada.

Los resultados fueron analizados con el software estadístico SPSS 1.1.

\section{Resultados}

En el período comprendido entre Enero de 2006 y Mayo de 2007 se practicaron 42 hernioplastías de urgencia que fueron ingresadas al protocolo de estudio por los cirujanos colaboradores del servicio de urgencia. Uno fue excluido por presentar importante contaminación fecaloídea producto de perforación colónica secundaria a compromiso vascular extenso en una hernia inguinal estrangulada.

Las características generales de la población estudiada se resumen en la Tabla 2 .

De los 41 pacientes estudiados, 6 presentaron cultivo positivo, en este caso para Staphylococcus coagulasa negativo en la muestra $\mathrm{N}^{\circ} 1$ (obtenida con tórula desde la superficie del saco). La muestra $\mathrm{N}^{\circ} 2$ (fragmento de saco herniario) resultó positiva para Streptococcus sp, en sólo un paciente portador de cirrosis alcohólica y abundante líquido libre en la cavidad abdominal.

La mayoría de los pacientes estudiados se encontraban al ingreso en buen estado general, afebriles, 3 de ellos presentaban eritema superficial en la zona comprometida, y 6 pacientes $(14,6 \%)$ presentaban signos peritoneales.

Entre los exámenes de laboratorio, el recuento de leucocitos promedio fue de $9.230 \mathrm{cel} / \mathrm{mm}^{3}(6.000-$
17.000) y la Proteína $\mathrm{C}$ reactiva promedio fue de $18,8 \mathrm{mg} / \mathrm{L}(1,4-125,9)$. Ocho de los pacientes presentaron compromiso vascular en el contenido herniario, de los cuales 2 requirieron de resección intestinal del asa comprometida luego de no recuperar vitalidad al liberar la estrangulación. El tiempo de evolución postoperatoria oscila entre 12 horas y 10 días.

Las características locales de la hernia se resumen en la Tabla 3.

Al 78\% de los pacientes estudiados se les efectuó hernioplastía con malla. Sólo 2 pacientes evolucionaron en forma tórpida con infección de la herida operatoria, respondiendo favorablemente al tra-

Tabla 2. Características generales de la población

\begin{tabular}{lcc}
\hline $\mathbf{n = 4 1}$ & & \\
\hline Sexo & & \\
$\quad$ Varón & 11 & $(27 \%)$ \\
Mujer & 30 & $(73 \%)$ \\
Edad media & 61,3 años (rango: 46-88) \\
Comorbilidad & & \\
$\quad$ Hipertensión & 25 & $(60 \%)$ \\
Diabetes & 5 & $(12,2 \%)$ \\
Asma & 2 & $(4,8 \%)$ \\
ASA & & \\
I & 6 & $(14,6 \%)$ \\
II & 17 & $(41,4 \%)$ \\
III & 16 & $(39 \%)$ \\
IV & 2 & $(4,8 \%)$ \\
V & 0 & \\
\end{tabular}

Tabla 3. Características locales de la población

\begin{tabular}{lrr}
\hline $\mathbf{n = 4 1}$ & & \\
\hline Tipo de hernia & & \\
$\quad$ Inguinal & 4 & $(9,8 \%)$ \\
$\quad$ Incisional & 15 & $(36,6 \%)$ \\
$\quad$ Crural & 12 & $(29,2 \%)$ \\
$\quad$ Umbilical & 10 & $(24,3 \%)$ \\
Contenido del saco & & \\
$\quad$ Epiplón & 34 & $(82,9 \%)$ \\
$\quad$ Int. Delgado & 19 & $(46,3 \%)$ \\
$\quad$ Colon & 4 & $(9,8 \%)$ \\
$\quad$ Yeyuno & 1 & $(2,5 \%)$ \\
Diámetro max. Saco & $12,2 \mathrm{~cm}(\mathrm{rango:} 2-20 \mathrm{~cm})$ \\
Compromiso vascular & & \\
$\quad$ Sí & 8 & $(19,5 \%)$ \\
$\quad$ No & 33 & $(80,5 \%)$ \\
\hline
\end{tabular}


tamiento antibiótico (uno de ellos con cultivo positivo en fragmento de saco). El resto no presentó complicaciones inmediatas en un plazo de 1 mes de seguimiento, incluidos los que presentaron cultivos positivos en muestra de superficie.

\section{Discusión}

La utilización de material protésico en la reparación de las hernias complicadas es un tema discutible. Diversos autores han probado la técnica obteniendo resultados similares en relación a la hernioplastía electiva $^{21-23}$.

No existen criterios definitivos que permitan apoyar o desestimar el uso de malla en estos $\operatorname{casos}^{24}$.

La presencia de bacterias en el sitio de implantación protésica en las hernias complicadas parece ser uno de los principales temores del cirujano al tomar la decisión, ya que el riesgo de complicación es altísimo por infección de la malla ${ }^{24,25}$. No obstante, no hay publicaciones que establezcan fehacientemente la colonización del saco herniario ni tampoco las características clínicas o de laboratorio que se asocian a la presencia de estos agentes.

Nuestros resultados permiten establecer, al menos en forma inicial, que la mayor parte de las hernias complicadas operadas en la unidad de emergencia no presentan translocación bacteriana al saco, lo que tiene muy buena correlación con las características clínicas y de laboratorio.

En el caso de los pacientes con cultivos positivos para Staphyloccocus coagulasa negativo, al cotejar este resultado con la muestra $\mathrm{N}^{\circ} 2$ (fragmento de saco), las condiciones clínicas y de laboratorio, así como la favorable evolución postoperatoria a 1 mes de seguimiento, nos hace pensar que probablemente no sea más que un falso positivo a raíz de contaminación de la muestra, puesto que no recibieron terapia antimicrobiana terapéutica. El único paciente con cultivo positivo para la muestra correspondiente a fragmento de saco herniario, evolucionó con infección de la herida operatoria, siendo dado de alta al décimo día de terapia antibiótica.

Cabe mencionar que nuestro estudio requiere de un mayor número de casos para establecer valores más confiables, lo que se logrará probablemente incluyendo el total de hernias complicadas operadas en urgencias.

En conclusión, se observa que casi la totalidad de las hernias complicadas en la unidad de emergencia no presentan contaminación bacteriana en el saco herniario.

Las condiciones clínicas y de laboratorio permiten inferir cuando el proceso inflamatorio no es extenso, siendo menor el riesgo de translocación.
La posibilidad de infección de la malla a partir de un foco de translocación bacteriana secundario a la incarceración herniaria es mínima.

Desde el punto de vista de la microbiología local del saco herniario en estas condiciones, la utilización de prótesis parece ser segura.

Resta estudiar, en nuestra población, los resultados de la hernioplastia protésica en hernias complicadas con un tamaño muestral mayor, de manera de establecer una significancia estadística confiable y de esa forma validar el uso de malla en estos cuadros de urgencia.

\section{Referencias}

1. Kingsnorth A, Leblanc K. Hernias: inguinal and incisional. Lancet 2003; 8: 1561-1571.

2. Luijedink RW, Hop WC, Van Den Tol MP, De Lange DC, Braaksma MM, Ijzermans JN, et al. A comparison of suture repair with mesh repair for incisional hernia. N Engl J Med 2000; 10: 343-392.

3. Scott NW, Mccormak K, Graham P, Go PM, Ross SJ, Grant AM. Open mesh versus non-mesh for repair of femoral and inguinal hernia. Cochrane Database Syst Rev 2002; 4: 91-97.

4. Bardavid C, Guzmán H, Albarrán V, Blake P, Rodríguez L, Alamo M, y cols. Seis años de estudio: 537 hernioplastias inguinales libres de tensión. Rev Chil Cir 2004; 56: 453-457.

5. Floody M, Rodríguez C, Hernández E, Leiva F. Hernioplastía inguinal con malla. Rev Chil Cir 1994; 46: 643-646.

6. Wantz G. Hernias de la pared abdominal. En: Schwartz, Principios de Cirugía. Santiago de Acahualtepec. Editorial McGraw-Hill Interamericana, 2000; 1689-1716.

7. Papaziogas B, Lazaridis CH, Makris J, Koutelidakis J, Patsas A, Grigoriou M, et al. Tensión-free repair versus modified Bassini technique (Andrews technique) for stranguled inguinal hernia: a comparative study. Hernia 2005; 9: 156-159.

8. Mcintosh A, Hutchinson A, Roberts A, Whiters H. Evidence-based management of groin hernia in primary care-a systematic review. Fam Pract 2000; 17: 442-447.

9. Haapaniemi S, Sandblom G, Nilsson E. Mortality after elective and emergency surgery for inguinal and femoral hernia. Hernia 1999; 4: 205-208.

10. Birolini C, Utiyama EM, Rodríguez AJ, Birolini D. Elective colonic operation and prosthetic repair of incisional hernia: does contamination contraindicate abdominal wall prosthesis use? J Am Coll Surg 2000; 191: 366-372.

11. Rettenbacher T, Hollervewer A, Macheiner P, Gritzmann N, Gotwald T, Frass R, et al. Abdominal wall hernias: cross-sectional imaging signs of incarceration determined with sonography. AJR Am J Roentgenol 2001; 177: 1061-1066. 
12. Iedioha U, Alani A, Modak P, Chong P, O'dweyr PJ. Hernias are the most common cause of strangulation in patients presenting with small bowel obstruction. Hernia 2006; 10: 1-3.

13. O'Boyle CJ, Macfie J, Mitchell CJ, Johnstone D, Sagar PM, Sedman PC. Microbiology of bacterial translocation in humans. Gut 1998; 42: 29-35.

14. Robinson TN, Clarke JH, Schoen J, Walsh MD. Major mesh-related complications following hernia repair: events reported to the Food and Drug Administration. Surg Endosc 2005; 19: 1556-1560.

15. Fawole AS, Chaparala RP, Ambrose NS. Fate of the inguinal hernia following removal of infected prosthetic mesh. Hernia 2006; 10: 58-61.

16. Aufenacker TJ, Koelemay MJ, Gouma DJ, Simons MP. Systematic review and meta-analysis of the effectiveness of antibiotic prophylaxis in prevention of wound infection after mesh repair of abdominal wall hernia. Br J Surg 2006; 93: 5-10.

17. Beltrán M, Cruces K, Tapia T, Vivencio A. Resultados Quirúrgicos de la hernioplastía de Lichtenstein de urgencia. Rev Chil Cir 2006; 5: 359-364.

18. Roselló J, Molina E, Canals P, Mederos O, Enríquez E. Uso de Bioprótesis en las hernias inguinocrurales com- plicadas. Rev Cubana Cir 2003; 42: 342-348.

19. Wysocki A, Pozniczek M, Krzywon J, Strsalka M. Lichtenstein repair for incarcerated groin hernias. Eur J Surg 2002; 168: 452-454.

20. Pans A, Desaive C, Jacquet N. Use of a preperitoneal prosthesis for strangulated groin hernia. Br J Surg 1997; 84: 310-312.

21. Wysocki A, Pozniczek M, Krzywon J, Bolt L. Use of polypropylene prostheses for strangulated inguinal and incisional hernias. Hernia 2001; 5: 105-106.

22. Hasan Abbas M. Outcome of strangulated inguinal hernia. Pak J Med Sci 2005; 21: 445-450.

23. Álvarez JA, Baldonedo RF, Bear IG, Solís JA, Álvarez P, Jorge JI. Incarcerated groin hernias in adults: presentation and outcome. Hernia 2004; 8: 121-126.

24. Bellón J. Mallas y cirugía. ¿Cuáles y cuándo? Cir Esp 2003; 74: 1-3.

25. Finan KR, Vick CC, Kiefe CI, Neumayer L, Hawn MT. Predictors of wound infection in ventral hernia repair. Am J Surg 2005; 190: 676-681.

26. Bliziotis IA, Kasiakou SK, Kapaskelis AM, Falagas ME. Mesh-related infection after hernia repair: case report of an emerging type of foreign-body related infection. Infection 2006; 34: 46-48. 